\title{
Proposal for Austere Light Attack Aircraft - Project Aardvark
}

\author{
Joseph V. Hayes ${ }^{1}$, Timothy A. Heath ${ }^{2}$, Brady J. Alexander ${ }^{1}$, Jorge Fernandez - Coppel Velasco ${ }^{1}$, Spencer L. Grady ${ }^{2}$, \\ Noah M. Jorgensen ${ }^{1}$, Joshua C. Richardson ${ }^{1}$, and Veronica A. Rodriguez ${ }^{2}$ \\ University of Alabama-Huntsville, Huntsville, AL, 35899
}

The WickedAir senior design team at the University of Alabama in Huntsville is developing the preliminary design of an aircraft for the AIAA Undergraduate Team Aircraft Design Competition. The RFP calls for an affordable light attack aircraft capable of executing missions currently only feasible with attack helicopters. The aircraft must be able to operate from short austere fields and accommodate a crew of two. Additional design goals include enhanced survivability and the ability for deploying a variety of weapons including an integrated gun for ground targets. The aircraft must accomplish an attack mission with a full weapons load and a long-range ferry mission with a $60 \%$ weapons load. Through evaluation of existing attack aircraft and helicopters and initial trade studies, the team has produced a conceptual design for the ZA-13 "Aardvark". The 12,000 lbf Aardvark has twin turboprop engines mounted on a 6.9 aspect ratio swept wing. Two sponsons offer weapons attachment points similar to those of a helicopter. This design offers low speed performance, a high payload capacity, and a short takeoff length. Specific consideration was given to the effects of foreign object debris and particulate matter pollution with regards to the lifespan and vulnerability of the aircraft in various austere environments. This paper summarizes the detailed design, cost analysis, and mission capabilities behind the current aircraft.

\section{Nomenclature}

$\begin{array}{ll}\mathrm{AIM} & =\text { Air intercept missile } \\ \mathrm{CAS} & =\text { Close air support } \\ \mathrm{C}_{\mathrm{D}, 0} & =\text { Zero lift coefficient of drag } \\ \mathrm{C}_{\mathrm{D}, \mathrm{i}} & =\text { Induced coefficient of drag } \\ \mathrm{CDR} & =\text { Concept design review } \\ \mathrm{C}_{\mathrm{fe}} & =\text { Coefficient of equivalent friction } \\ \mathrm{CG} & =\text { Center of gravity } \\ \mathrm{ConOps} & =\text { Concept of Operations } \\ \mathrm{CPFH} & =\text { Cost per flying hour } \\ \text { DoD } & =\text { Department of Defense } \\ \mathrm{ESHP} & =\text { Equivalent shaft horsepower } \\ \mathrm{FOD} & =\text { Foreign object or debris } \\ \mathrm{GATR} & =\text { Guided advanced tactical rocket } \\ \mathrm{GBU} & =\text { Guided bomb unit } \\ \mathrm{HoQ} & =\text { House of Quality } \\ \mathrm{L} & =\text { Rib spacing } \\ \text { LLT } & =\text { Lifting line theory } \\ \mathrm{M}_{\max } & =\text { Maximum bending moment } \\ \text { MTOW } & =\text { Maximum take-off weight } \\ \mathrm{q}_{\infty} & =\text { Dynamic pressure }\end{array}$

${ }^{1}$ Aerospace Engineering Senior, Mechanical and Aerospace Engineering, AIAA Student Member

${ }^{2}$ Mechanical Engineering Senior, Mechanical and Aerospace Engineering, AIAA Student Member 


$\begin{array}{ll}\text { PGM } & =\text { Precision-guided munition } \\ \mathrm{R}_{\max } & =\text { Maximum resultant force } \\ \mathrm{RFP} & =\text { Request for proposal } \\ \mathrm{S} & =\text { Planform area } \\ \mathrm{S}_{\text {wet }} & =\text { Total wetted area } \\ \mathrm{V} & =\text { Velocity } \\ \mathrm{V}_{\max } & =\text { Maximum shear force } \\ \mathrm{VLM} 1 & =\text { Horseshoe vortex analysis } 1 \\ \mathrm{VLM} 2 & =\text { Ring vortex analysis } 2 \\ \text { Del } & =\text { Approximate circumference of airfoil } \\ \sigma_{\text {yield }} & =\text { yield axial stress } \\ \tau_{\text {yield }} & =\text { yield shear stress }\end{array}$

\section{Introduction}

The WickedAir senior design team at the University of Alabama in Huntsville is competing in the 2020/2021 AIAA Undergraduate Team Aircraft Design Competition. As stated in the Request for Proposal (RFP), "The objective of the project is to design an affordable light attack aircraft that can operate from short, austere fields near the front lines to provide close air support to ground forces at short notice and complete some missions currently only feasible with attack helicopters." [1] In addition to a designated project lead and chief engineer, the design team was organized to address the primary technical areas of aerodynamics, propulsion, structures, stability and control, avionics, and mechanical systems. To address specific requirements in the RFP, team members were also assigned to cost analysis and human factors. The team completed a conceptual design where a database of similar aircraft serving in a close air support (CAS) role was compiled and evaluated. An alternatives selection process helped define the initial concept. The team is currently performing preliminary design trade studies to refine the concept. This paper describes the design process to date and the status of the team's concept - the ZA-13 “Aardvark".

\section{A. Requirements and Objectives}

\section{Project Definition}

The AIAA RFP provides a list of requirements and objectives that are shown in Table 1. Here, [R] designates a mandatory requirement and [O] designates a goal or desired objective. Most of the mandatory requirements include actual quantitative metrics to satisfy. The objectives tend to be general goals and the design team had to define metrics that will attain these requirements.

\section{Table 1. AIAA RFP Requirements and Objectives}

\begin{tabular}{|c|l|c|}
\hline 1 & $\begin{array}{l}\text { Austere Field Performance: Takeoff and landing over a } 50 \mathrm{ft} \text { obstacle in } \leq 4,000 \mathrm{ft} \text { when operating } \\
\text { from austere fields at density altitude up to 6,000 ft with semi-prepared runways such as grass or } \\
\text { dirt surfaces with California Bearing Ratio of } 5\end{array}$ & $\mathrm{R}$ \\
\hline 2 & $\begin{array}{l}\text { Survivability: Consideration for survivability, such as armor for the cockpit and engine, reduced } \\
\text { infrared and visual signatures, and countermeasures (chaff, flares, etc.) }\end{array}$ & $\mathrm{O}$ \\
\hline 3 & Payload: 3000 lbs of armament & $\mathrm{R}$ \\
\hline 4 & $\begin{array}{l}\text { Provisions for carrying/deploying a variety of weapons, including rail-launched missiles, rockets, } \\
\text { and 500 lb (maximum) bombs }\end{array}$ & $\mathrm{O}$ \\
\hline 5 & Integrated gun for ground targets & $\mathrm{R}$ \\
\hline 6 & Service life: 15,000 hours over 25 years & $\mathrm{R}$ \\
\hline 7 & Service ceiling: $\geq 30,000 \mathrm{ft}$ & $\mathrm{R}$ \\
\hline 8 & Crew: Two, both with zero-zero ejection seats & $\mathrm{R}$ \\
\hline
\end{tabular}

The RFP also defines a design mission and a ferry mission. The design mission represents deployment of the aircraft to a combat zone for patrolling and potentially engaging enemy targets. This mission emphasizes the aircraft's speed, maneuverability, and loiter (endurance) capabilities. The long-range ferry mission is a redeployment mission in which the aircraft must carry 60 percent of its max payload weight at least 900 nautical miles. 
Based on the stated desire to fulfill some missions currently performed by helicopters, the team conducted research on what advantages and disadvantages helicopters provide against fixed-wing aircraft. Helicopters have a very small loitering area, can achieve acoustic and visual terrain masking, can fly at low altitudes and require no runway. However, they are limited to slower cruise speeds, are relatively complex, and typically cannot deploy bombs. Considering these characteristics of helicopters, the design team placed an emphasis on the ZA-13 Aardvark being able to operate at very low stall speeds and small loiter areas.

\section{B. Concept of Operations}

Table 1 shows specific requirements for the aircraft. The RFP also defines mission profiles for the design mission and ferry mission. Figure 1 illustrates the Concept of Operation for the two missions. From these mission profiles we can derive additional requirements and goals for our design. Because the takeoff altitude is at a maximum of 6,000 feet, we can determine the minimum density (ignoring humidity effects of density altitude) during takeoff to be $0.0019869 \mathrm{slug} / \mathrm{ft}^{3}$. We also know from the California bearing ratio that the rolling friction coefficient of the landing gear will reliably be around 0.8 to 1.0 . Additionally, from the design mission the maximum endurance of the aircraft must amount to more than five and a half hours. A goal for maximum velocity of $300 \mathrm{kts}$ can also be obtained from the design mission cruise phase.
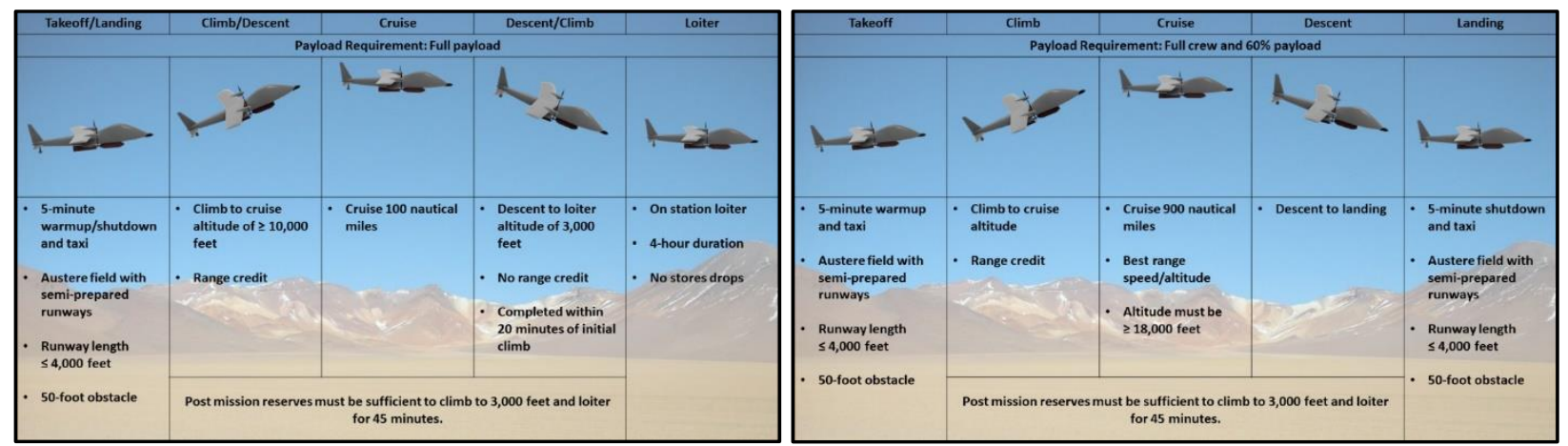

Fig. 1. Design Mission (left) and Ferry Mission (right) ConOps

\section{House of Quality}

A House of quality was developed to organize and prioritize the customer requirements and identify design features that would help meet these requirements. The House of Quality in Fig. 2 shows our assessment or the customer's priorities and the relative importance of different parts of our design.

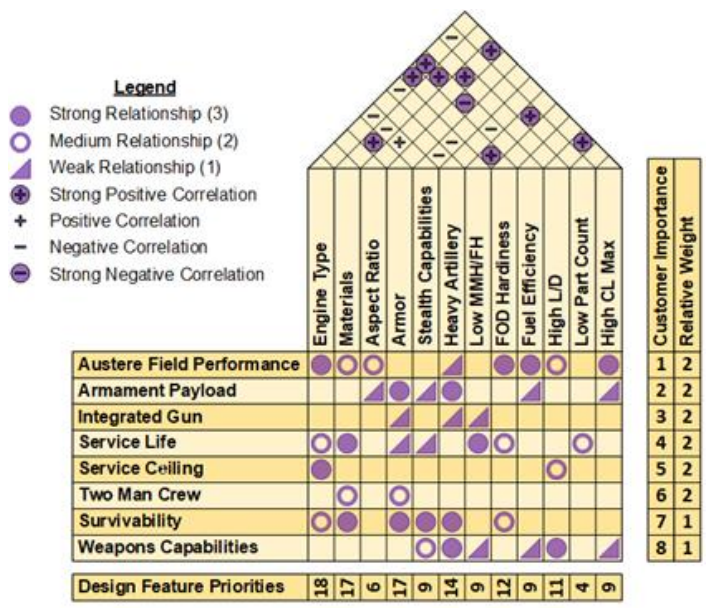

Fig. 2 House of Quality for Light Attack Aircraft 


\section{Concept Selection}

The starting point of a design can be critical in how long a project takes and the success of the end result. As such, a characteristic database of historical and current light attack aircraft and helicopters was compiled, configuration alternatives were evaluated, and initial trade studies on multiple concept designs were performed. Approximate characteristics derived from this process include a 310 knot max speed, 32,000 ft service ceiling, $200 \mathrm{ft}^{2}$ planform area, an aspect ratio of 6, and 12,000 lb MTOW. Three candidate conceptual designs were defined: the Emu (Fig. 3), the Aardvark (Fig. 4), and the twin fuselage Guppy (Fig. 5).
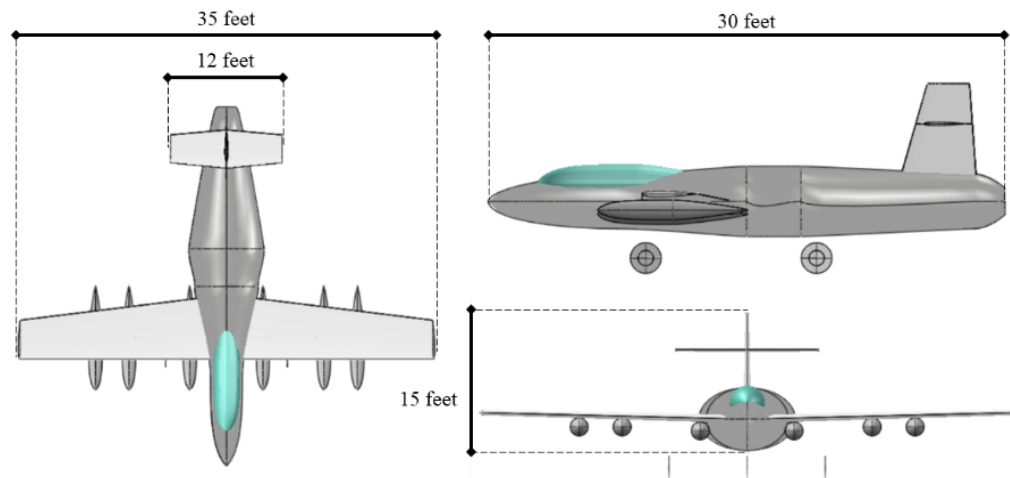

(C)
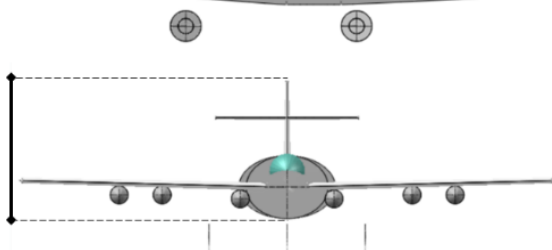

Fig. 3. Design Concept 1 - Emu

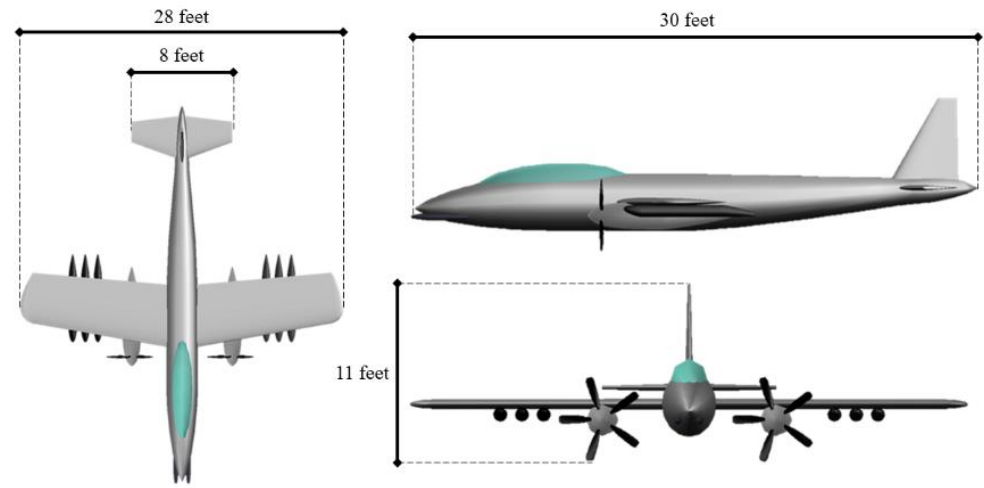

Fig. 4. Design Concept 2 - Aardvark

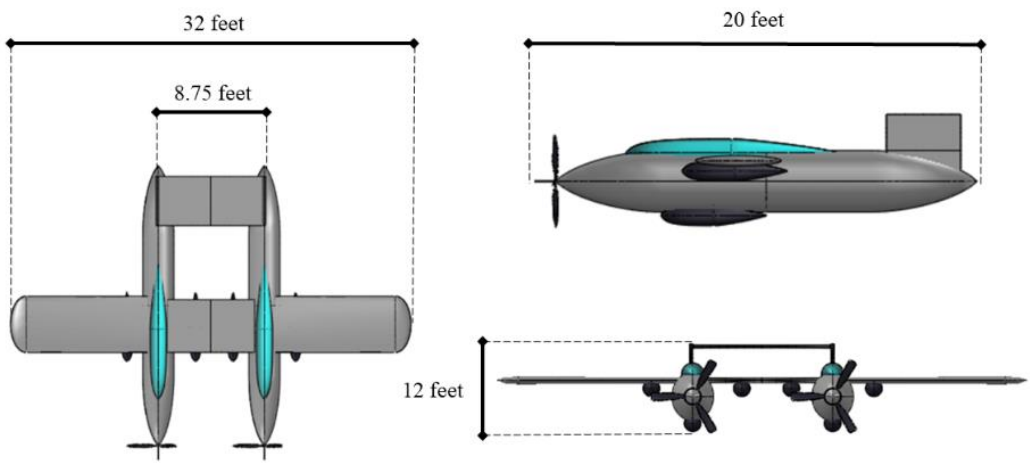

Fig. 5. Design Concept 3 - Gupy 
One of the most important decisions is choosing a propulsion system. The Aardvark and Guppy utilize turboprop engines and the Emu uses a turbofan engine. The reason for this is because Fig. 5 below shows engine family operation spaces in terms of service ceiling and flight speed. The turboprop and turbofan engines can meet our service ceiling and speed requirements. A turbojet can also meet our requirements but is a much less efficient alternative.

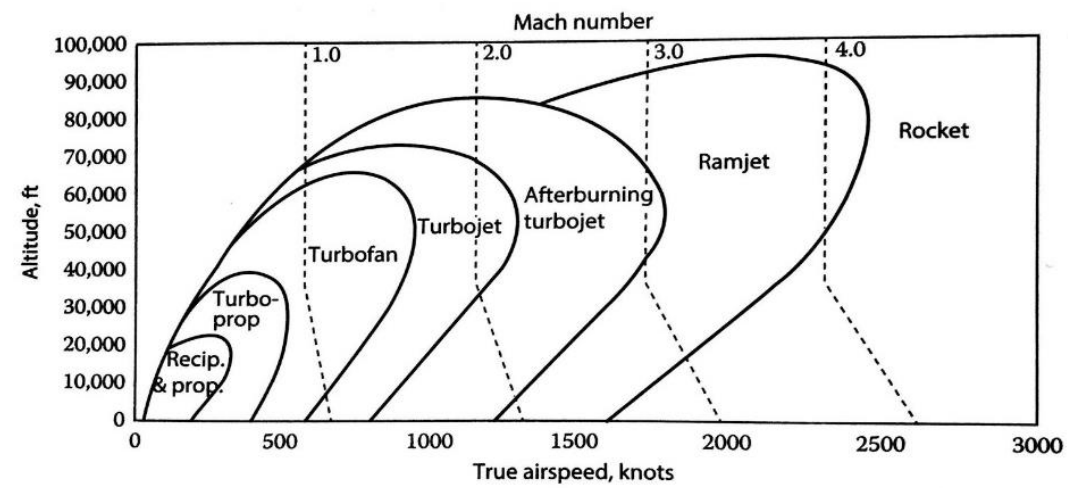

Figure 5: Aircraft Propulsion System Operating Envelopes [2]

A set of 6 key design factors, shown in Table 2, were evaluated for each aircraft. Of the 6 criteria, FOD survivability, Engine type, and combat survivability were directly influenced by our House of Quality. Of the three proposed solutions, the Aardvark was selected as our team's starting concept design primarily due to the FOD hardiness and maintenance advantages of a turboprop compared to a turbofan. Additionally, two of engines offer redundancy if one were to fail or be damaged mid-flight. The single fuselage is also much more stable compared to a twin fuselage arrangement according to pilot reports from similar aircraft.

Table 2. Concept Design and Selection Trade Study

\begin{tabular}{|c|c|c|c|c|c|c|}
\hline & $\begin{array}{c}\text { Engine } \\
\text { Class }\end{array}$ & $\begin{array}{c}\text { \# of } \\
\text { Engines }\end{array}$ & $\begin{array}{c}\text { Combat } \\
\text { survivability }\end{array}$ & $\begin{array}{c}\text { FOD } \\
\text { survivability }\end{array}$ & Stability & Expected cost \\
\hline Emu & Turbofan & 1 & Low & Bad & High & High \\
\hline Aardvark & Turboprop & 2 & High & Good & Medium & Low \\
\hline Twin Fuselage & Turboprop & 2 & Medium & Good & Low & Medium \\
\hline
\end{tabular}

\section{Design Overview}

\section{A. Geometry}

Since the selection of Design Concept 2 - Aardvark, several revisions have been made. The current configuration is shown in Fig. 7. The 12,000 lbf Aardvark has twin turboprop engines mounted on a 6.9 aspect ratio swept wing. Two sponsons offer weapons attachment points like those of a helicopter. This design offers low speed performance, a high payload capacity, and a short takeoff length. Subsequent sections will describe several design details. 


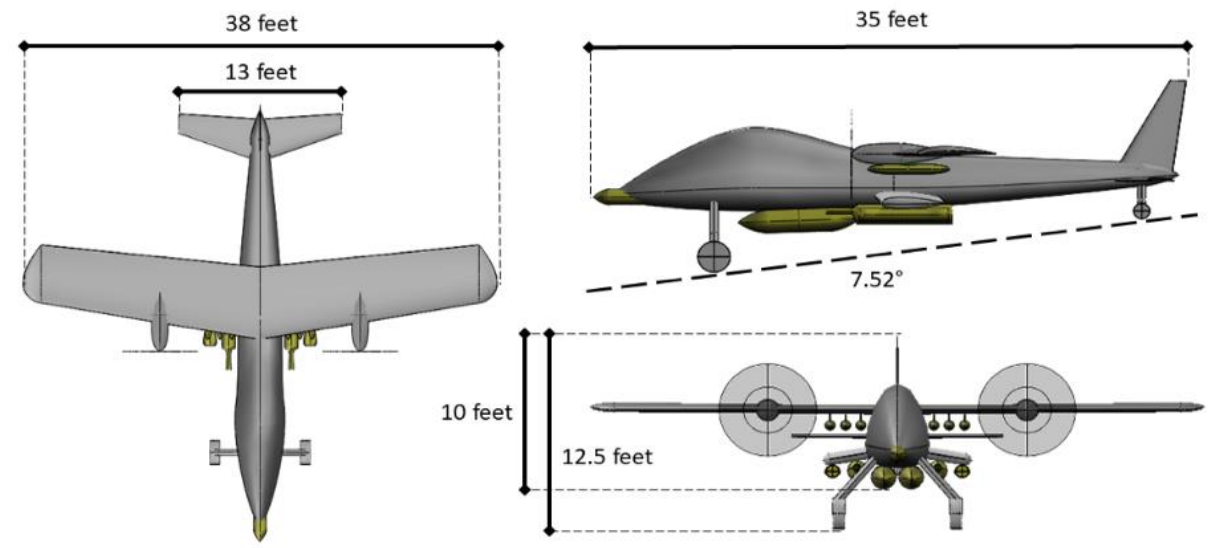

\section{B. Wing Design}

Fig. 7. Aardvark Three View Sketch

The wing must be designed to support engine and armament loads, generate adequate lift at low speeds, and withstand high speed and maneuvering stresses. A NACA 4412 airfoil was chosen for the current design due to its high maximum lift coefficient to provide a low stall speed, and a trailing edge stall. The $12 \%$ thickness also accommodates internal wing supports needed to support the wing loads and counteract maneuvering stresses.

The current design for the wing can be seen below in Fig. 8. For the purposes of a better understanding of the wings aerodynamic performance and gathering useful data for structural analysis, the wing was simulated in XFlr5 [2] that uses a vortex lattice method coupled with the Xfoil airfoil analysis. We decided to use a panel count of 20 in the chord direction and 40 in the span direction were used. A 280 knots flight velocity and $3.5^{\circ}$ angle of attack were calculated as the parameters for our dive bomb run in the combat patrol phase of the design mission. Fig. 9 shows the predicted wing lift, viscous drag, and induced drag distributions for these inputs. The predicted lift of 30,000 lbf is consistent with the approximately $29,000 \mathrm{lbf}$ of lift predicted by hand calculations based on the aircraft weight, $\mathrm{G}$ forces, and lift coefficient estimations.

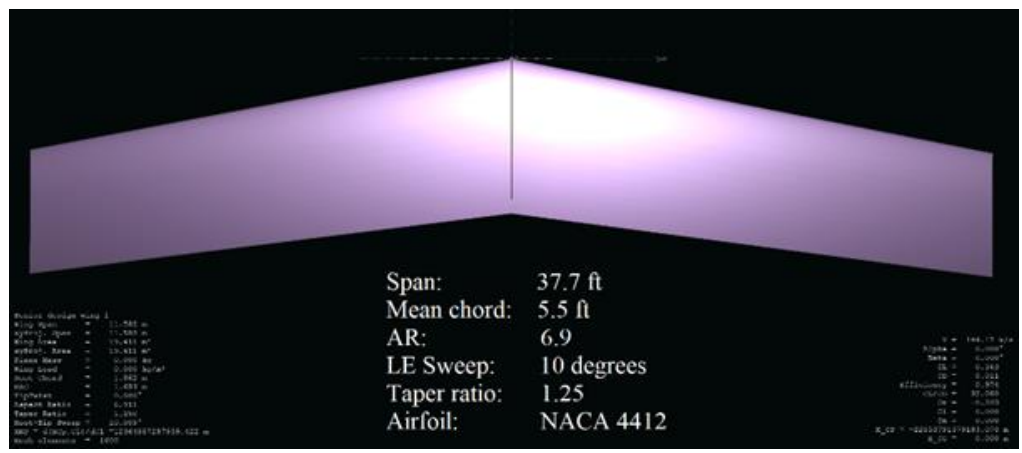

Fig. 8. Current Wing Design Model in Xflr5 [REF] 


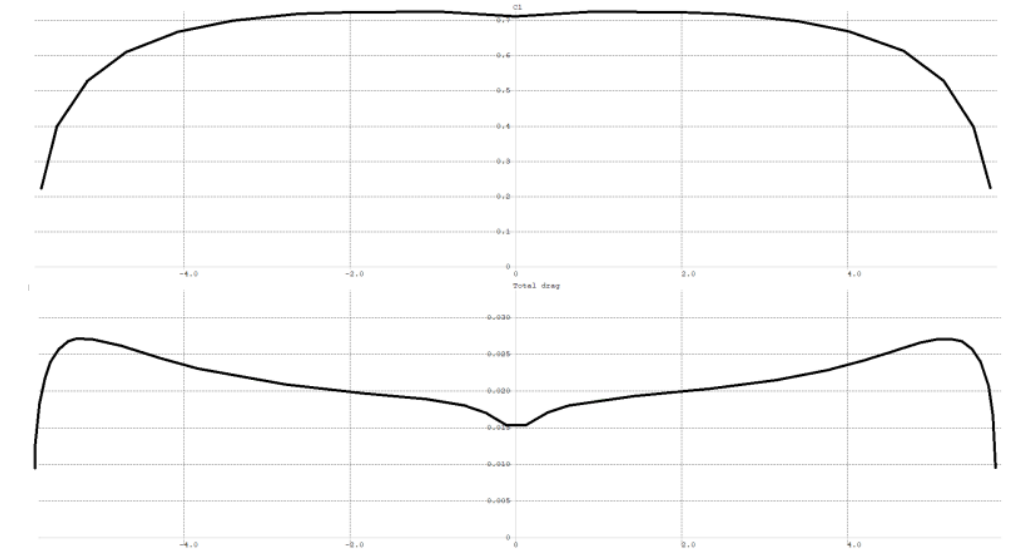

Fig. 9. Xflr5 predicted wing lift (top) and drag (bottom) distributions for simulated dive bomb run

The Xflr5 predicted lift, drag, and bending moment distributions will be used by the structures group for spar design and rib spacing. To this point, only the clean wing has been considered. The next major step is to determine the effects of the payload, propellers, and sponsons on lift and drag generation. These more in-depth analyses will likely impact our structural design and possibly our future wing design. As of now, the team assumes a significant decrease in lift and increase in drag.

\section{Structural Design and Analysis}

The structural analysis begins with a skeleton design, shown schematically in Fig. 10 from Ref. 2, and basic materials selection. Our current materials selection trade study only covers the primary wing structure. We have selected materials for the wing skin, ribs, and spars. Each of these components has different priorities and as such different materials in the study and selection.

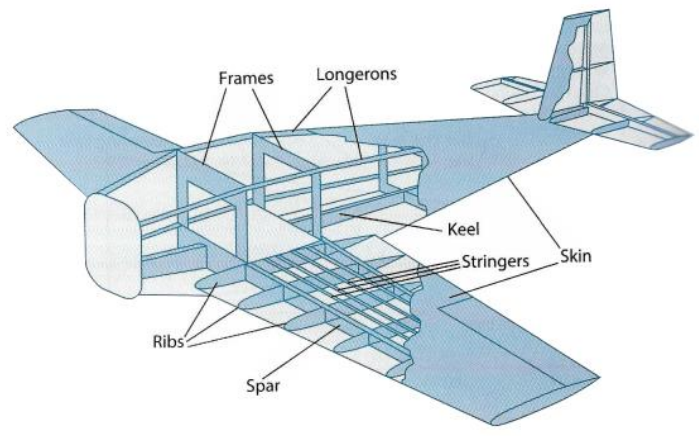

Fig. 10. Types of Structural Components [3]

During a mission, the aircraft will experience high aerodynamic and inertial loading. The loading may also be cyclical. Spars are the main structural member of the wing and must carry the largest loads. Therefore, the material used for the spars should be strong and resistant to fatigue and fracture; but it should also be lightweight and machinable. Various forms of titanium, steel, and aluminum were examined for these qualities and ultimately, aluminum 7075-T6 was chosen for the spars due to its low density, high strength, and good machinability. It is not the cheapest aluminum alloy but the one with the most suitable mechanical properties for this application. Considering the spars are the main structural component of the wing, the cost is more than justified.

The load is transmitted from the wing skin though the ribs and to the spars. The ribs also provide the wing shape. Like the spars, steel, titanium, and aluminum were considered. A 6061-T6 aluminum was chosen for the rib material. Although 6061 aluminum has slightly lower strength compared to 7075 aluminum, 6061 aluminum is far more compatible with modern machining and manufacturing techniques and is still very strong.

The biggest threat to the skin is from bullets and FOD damage. Therefore, the skin needs to have very good fracture and fatigue resistant properties. It must also be very stiff and capable of withstanding heavy shear loads. The current strength, weight and cost estimations are based on using 2024-T3 for the skin. If the cost is not prohibitive, the team is evaluating incorporating a honeycomb structure consisting of carbon and aluminum; with an aramid paper as adhesive for insulation purposes in case there is a fire. 
Two main spars will be used as well as a uniform rib spacing. From the lift and drag distribution data we calculated a maximum resultant force of $760 \mathrm{lbf}$ very near the root. For a first order rib spacing calculation, we assumed that the skin is subjected to a uniform pressure distribution which totals to this resultant force. If we treat the skin of the aircraft like a beam fixed on both ends (see Fig. 11), the maximum bending moment and maximum shear can be calculated by Eqs. (1) and (2). Here, $\mathbf{M}_{\max \text {,Skin }}$ and $\mathrm{V}_{\mathrm{max} \text {,Skin }}$ are the maximum bending moment and shear force that the airplane skin can withstand, respectively. Additionally, $\mathrm{t}$ is the skin thickness and $\delta$ is the approximate cross-sectional circumference. We also use a factor of safety of 1.5 for the pressure distribution magnitude. Assuming Aluminum 2024-T3 for the skin, the maximum axial stress is calculated to be $47 \mathrm{ksi}$ and the maximum shear stress is calculated to be $41 \mathrm{ksi}$.

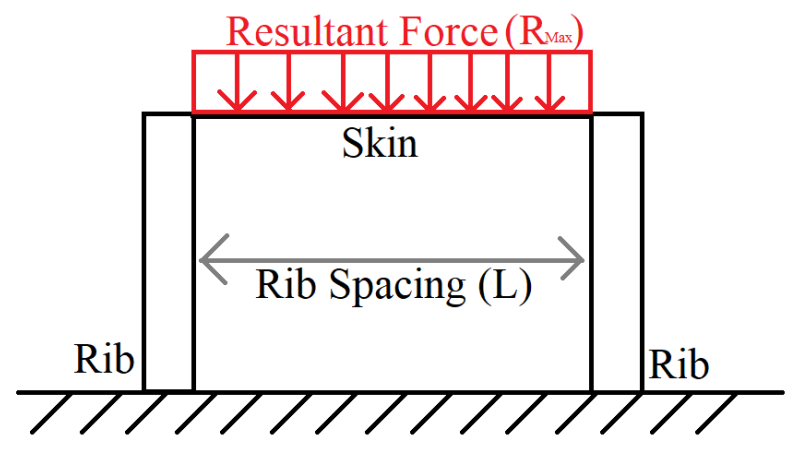

Fig. 11. Rib Calculation Simplification Reference

$$
\begin{gathered}
\mathrm{M}_{\text {max,skin }}=\frac{\mathrm{R}_{\text {max }} \mathrm{L}^{3}}{16}=\frac{\sigma_{\text {yield }} \mathrm{t}^{2} \delta}{6} \\
\mathrm{~V}_{\text {max,skin }}=0.75 \mathrm{R}_{\text {max }} \mathrm{L}=\tau_{\text {yield }} \mathrm{t} \delta
\end{gathered}
$$

After solving for the rib spacing, we calculate a maximum spacing of $3.22 \mathrm{ft}$. The wing is 37.7 feet in span which results in us requiring 12 ribs in total. This number also assumes ribs in the fuselage, so for weight and cost estimates the two middle ribs can be ignored. The rib spacing calculation will need to be refined. In addition, an analysis of the torsion and root bending moment underwing loading must be determined to assess the strength of the wing design.

\section{Propulsion and Performance}

The propulsion analysis started with a survey of engine families that might be compatible with our design. This yielded the Honeywell TPE331 series and the Pratt-Whitney PT6A series. Both families offer several engines at different thrust levels to select from while still providing good performance and reliability. The initial engine that was selected is the Pratt-Whitney PT6A-25C. The engine specifications are shown in Table 3.

Table 3. Pratt-Whitney PT6A-25C Specifications

\begin{tabular}{|c|c|}
\hline US Military Designation & T74 \\
\hline Takeoff ESHP & 783 ESHP \\
\hline Approximate weight & $355 \mathrm{lbs}$ \\
\hline Approximate dimensions & 62 ” $\times 23$ ” \\
\hline Overhaul Interval & $3000 \mathrm{hrs}$ \\
\hline Specific fuel consumption & $0.595 \mathrm{lb} / \mathrm{hp} \cdot \mathrm{hr}$ \\
\hline
\end{tabular}

Fig. 12 shows the power required and power available curves at a cruising altitude of 10,000 feet. The power available curve was obtained by using the maximum rated continuous power provided by both engines and an assumed 
$85 \%$ propeller efficiency. The power required curve was calculated using Eq. 3 . The $C_{D, 0}$ value used in Eq. 3 is calculated by using the concept of equivalent skin-friction drag coefficient, $\mathrm{C}_{\mathrm{fe}}$, where drag data has been compiled for various categories of aircraft to allow for an average $\mathrm{C}_{\mathrm{fe}}$ value to give a reasonable estimate of $\mathrm{C}_{\mathrm{D}, 0}$, as seen in Eq. 4. The total wetted area of the aircraft, $S_{\text {wet }}$, was calculated by our CAD model.

$$
\begin{aligned}
\text { Power }_{\text {required }} & =\mathrm{Sq}_{\infty}\left(\mathrm{C}_{\mathrm{D}, 0}+\mathrm{C}_{\mathrm{D}, \mathrm{i}}\right) \mathrm{V} \\
\mathrm{C}_{\mathrm{D}, 0} & =\mathrm{C}_{\mathrm{fe}} \frac{\mathrm{S}_{\mathrm{wet}}}{\mathrm{S}}
\end{aligned}
$$

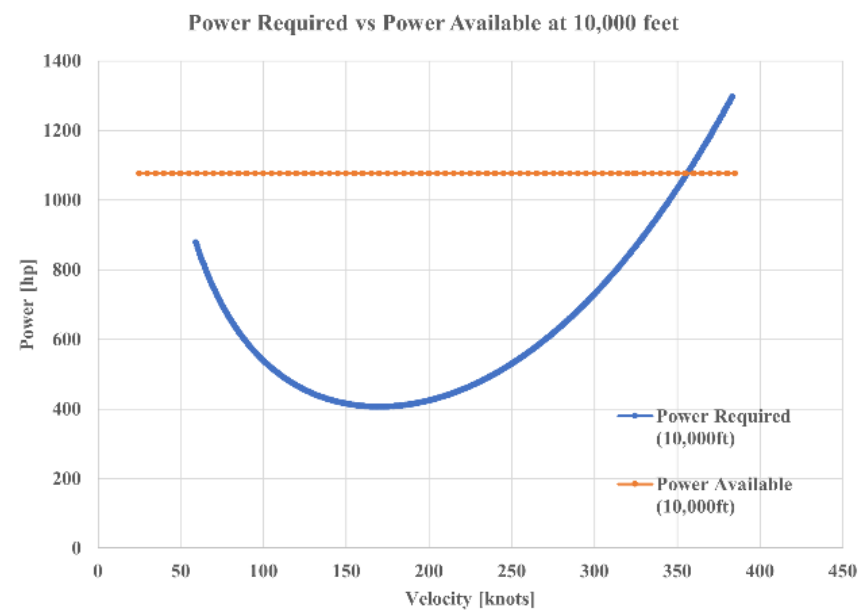

Fig. 12. Power Curves for the Aardvark

\section{E. Weapons}

The aircraft design should be capable of carrying/deploying at least $3000 \mathrm{lbf}$ of weapons including rail-launched missiles, rockets, and $500 \mathrm{lb}$ (maximum) bombs. The two weapons hardpoint configurations being considered for the aircraft are shown in Figure 13. The first configuration would carry the munitions underneath sponsons and the fuselage with two additional hardpoints, one under each wing. The other configuration would not have sponsons. There would be three hardpoints under each wing with one underneath the fuselage. Both configurations could carry the required $3000 \mathrm{lbs}$. The final weapons load configuration chosen will be based on aerodynamic and structural analysis of each.

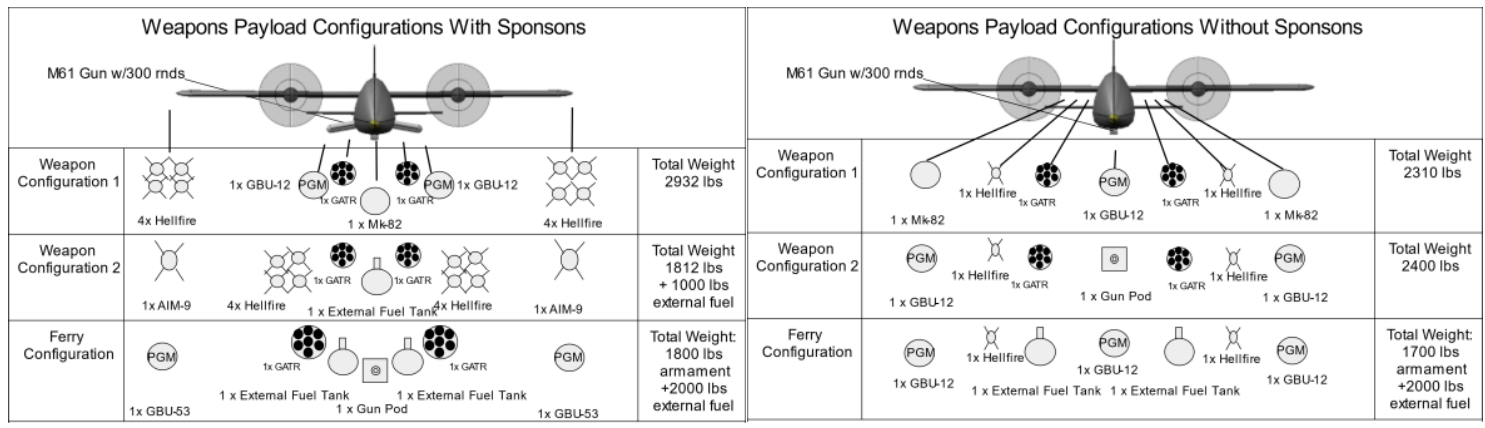

Fig. 13. Weapons Configurations with sponsons (left) and without sponsons (right)

\section{Cost Analysis}

Affordability is a very important design factor. Not only does the RFP specifically ask for an affordable design, but it goes without saying that light attack aircraft do not need to be nearly as advanced as multi-role fighter jets. The team collected data on the operating cost per hour of various aircraft. Figure 14 is a plot of the operating Cost per Flying Hour (CPFH) vs. the MTOW of the aircraft surveyed. Below you can see our cost analysis database. A linear regression was fit to the data and is given by Eq. (4). 


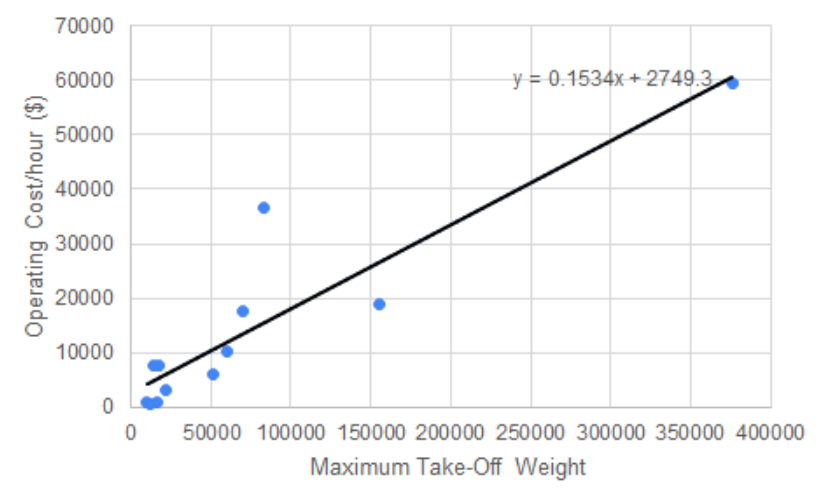

Figure 14: CDR phase operating cost analysis database

$$
\text { Cost } \approx 0.1534 * \text { MTOW }+2749
$$

The DoD uses CPFH to relate costs of aircraft to each other because it applies no matter the usage rate of the aircraft. However, one disadvantage is that it does not accurately represent the fixed costs of the aircraft. For the analysis of our aircraft, a hybrid version of CPFH will be used that is called CPFH (cross-system). This version of CPFH considers the fixed costs of an aircraft and CPFH (reimb). This does not take into consideration labor costs, salary of crew members, or any other cost that is not specifically dealing with the physical aircraft. Equations (6) and (7) are used in this calculation.

$$
\begin{gathered}
\text { CPFH }=\frac{\text { Fuel }+ \text { Consumables }+ \text { DLRs }}{\text { Flying Hours }} \\
\text { CPFH }(\text { reimb })=\mathrm{CPFH}+\frac{\text { Depot Maintenence }+ \text { Variable CLS }}{\text { Flying Hours }} \\
\mathrm{CPFH}(\text { Cross System })=\mathrm{CPFH}(\text { reimb })+\frac{\text { Fixed Cost }}{\text { Flying Hours }}
\end{gathered}
$$

By using the CPFH (cross-system) we can account for the fixed costs, which is a weakness of the original CPFH equation. To determine the value of CPFH (reimb) of our design, a trade study was performed comparing the CPFH (reimb) values of aircraft that are similar in design to our own. By comparing the CPFH (reimb) values of multiple fighters currently or recently used in combat, we noticed that the most common values were between $\$ 6,000$ and $\$ 10,000$. In our case, the only fixed cost that we need to consider is the cost of materials. It should be noted that the cost of fuel, consumables (tires, brakes, etc.) are included in the CPFH formula. Maintenance costs are included in the $\mathrm{CPFH}$ (reimb) formula to keep an accurate track of maintenance costs per flying hour.

Through our trade study, we were able to determine the average cost for aluminum is $\$ 0.30$ per pound. Using an estimated maximum takeoff weight of $12,000 \mathrm{lbf}$, it is estimated that the fixed cost per flying hour will be $\$ 4,500$. Therefore, the estimated CPFH (cross-system) of our current design is $\$ 10,500-\$ 14,500$. This would mean that for 1200 flying hours, the cost of the aircraft would range between $\$ 12.6$ million - $\$ 17.4$ million. Also, for design lots of 50 aircraft to manufacture the aircraft the cost would be estimated to be between $\$ 525,000$ - $\$ 725,000$ per aircraft, or $\$ 26.25$ million - $\$ 36.25$ million for 50 aircraft. This range is on par with current military aircraft and meets the expectations of affordability described in the RFP.

\section{Next Steps}

As we finalize our aerodynamics model, analysis of the static and dynamic stability can begin. This will include calculations of the CG window, and inclusion of the effect of downwash on the horizontal stabilizer. Sizing of the control surfaces must ensure good control authority for the pilot. As the wing structure is finalized, the fuselage skeleton will be designed. These cross-section shapes will be used to determine fuselage effects on the longitudinal moment for our stability analysis and allow cockpit design. The cockpit design will accommodate the required crew 
of two and provide ample visibility and protection. We will also incorporate zero-zero ejection seats. Another major focus is the FOD hardiness of the engines, skin, and landing gear. It is highly likely that the tail dragger design will be swapped for a nose wheel gear arrangement. More study of the sponsons needs to be performed to see if they provide a net benefit to the design performance. The sponsons will increase the aerodynamic drag. Although they may also provide a small addition to the lift, their location along the fuselage may also change the aerodynamics at various angles of attack. The sponsons would also be located where the rear wheels of a tricycle gear system would be located. If the required volume is feasible, the gear might be retracted into the sponsons. The current location places the sponsons near the aircraft center of gravity so that there is minimal movement when bombs are dropped. Moving the sponsons may have a detrimental effect on the aircraft balance and moments.

\section{Conclusion}

The current concept design meets many of the RFP requirements and is a good starting point for the continued design of WickedAir's project Aardvark. During upcoming months this design will be scrutinized, and changes will be implemented to meet both mission and design objectives, as well as customer needs; with the objective of optimizing design and performance making use of an iterative design process. This iterative process may include changes to features such as the horizontal stabilizer position, the sponson location, wing dihedral, mounting hardpoints, landing gear arrangement, etc. However, the concept level configuration will remain like as shown here today.

\section{Acknowledgments}

All the authors wish to thank David "Dutch" Ferguson, Lt Col, USAF (Retired) and John Schroeder, Maj, USAF (Retired) for providing their expertise and insight into the close air support mission.

\section{References}

[1] “AIAA 2021 Undergrad Team Aircraft Design RFP,” AIAA RFP

[2] XFLR5, Ver 6.48, MIT, 2019.

[3] Brandt, Steven A., Stiles, Randall J, Bertin, John J, and Whitford, Ray, Introduction to Aeronautics: A Design Perspective, $3^{\text {rd }}$ ed, AIAA Education Series, AIAA, Virginia, 2015.

[4] Join Chiefs of Staff, Joint Publication 3-09.3: Joint Tactics, Techniques, and Procedures for Close Air Support (CAS), Joint Chiefs of Staff, Washington, DC., 2003.

[5] United States, Department of Transportation, Federal Aviation Association. Type Certificate Data Sheet E4EA.

[6] Parker, Joel. "Use of a Common Assessment Methodology in Support of Joint Training, Capability Development, and Experimentation," US Joint Forces Command, 2007.

[7] McAndrew, Anne. "Fiscal Year (FY) 2019 Department of Defense (DoD) Fixed Wing and Helicopter Reimbursement Rates," Office of the Secretary of Defense, Washington, DC, 2018. 